\title{
Renewable Energy Fed Bidirectional Converter Based on Grid Application Using Fuzzy Logic-PID Controller
}

\author{
Kalluri Venkateswarlu' ${ }^{1}$ Dr. M. S. Godwin Premi ${ }^{2}$ \\ ${ }^{1}$ Research Scholar, Sathyabama University. \\ ${ }^{2}$ Professor, School of Electrical Engineering, Sathyabama University.
}

Article History:Received:11 november 2020; Accepted: 27 December 2020; Published online: 05 April 2021

\begin{abstract}
Photovoltaic systems containing rechargeable batteries as a power buffer takes extensive DC-DC converters for photovoltaic panel control and battery regulation. This paper suggests the non-isolated bidirectional fed grid for stand-alone pv systems in order to simplify the operation by reducing the number of transformers. In the bidirectional converter suggested, the power is transmitted from the solar to the grid. Then lack of PV power; back up storage that is battery supplies power. Fuzzy PID control is used to controls the voltage of DC along with increases the gain of voltage by means of a bidirectional converter. The proposed bidirectional converter implemented and the simulations are observed in MATLAB / Simulink.
\end{abstract}

Keywords: PV panel. Grid, Bidirectional converter, Fuzzy logic, PID controller, Battery.

\section{Introduction:}

Rechargeable batteries for stable power supplies, by tampering fluctuating solar energy production, are required for renewable energy systems such as PV systems. Multiple dc-dc converters, not only for panels, but also for batteries, are required in such systems [1-2]. In the use of electronic power converters has been growing in recent days and the bidirectional converter has been more appealing [3-5]. In medium to wide voltages the bidirectional inverter is used. The transformer is more appealing with galvano insulation and uses the pulsation width modulation to control the current of the dc link [6-7]. To preserve the strength of the bidirectional inverter, the angle of phase shift from primary into the secondary controls verified. Many industries attracted effective traditional PID controller by the simple simulation, flexibility modification and regulation. The fuzzy logic is applied and its expert structures are used to structure the operating points to improve accuracy [8-10]. The other controller like SMC and MPC has a lot of advantages over dynamic control, but it is very complicated to build and execute. The combined controller framework has now been created. The key goal of the architecture is to leverage all the advantages of the controls [11-12]. Due to its simplified structure and its high efficiency, the fuzzy-PID attracted most scientists in steady condition, and high performance in complex conditions benefited [13-14].

\section{Proposed Method:}

Instead of two unidirectional converters, a two-way dc-dc invert mechanism attached to the grid is used to pass electricity in both the forward and the reverse directions. Figure 1 displays the planned block diagram. The battery stores reverse power and serves as a supply while there are no photovoltaic photovoltaics in electric car applications. In order to reduce the existing battery ripple, the proposed solution would. The PV-connected grid inverter must reduce the overall total harmonics distortion (THD) in those charging currents along with increases about to power factor adjustment. The fuzzy-PID control is used to control the dc attach voltage in bidirectional converter. Then storage in battery-based generation of photovoltaic electricity is supplied to a bidirectional converter connected to the grid.

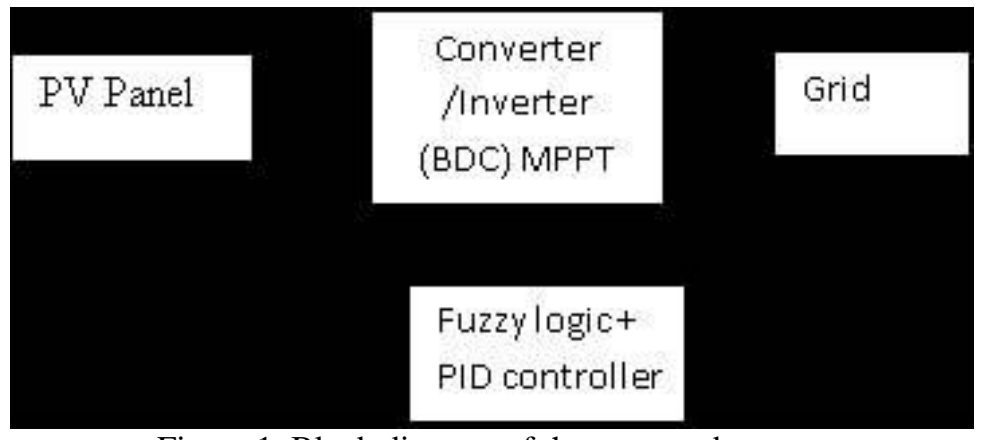

Figure 1. Block diagram of the proposed system

*Corresponding author: Kalluri Venkateswarlu

Research Scholar, Sathyabama University 


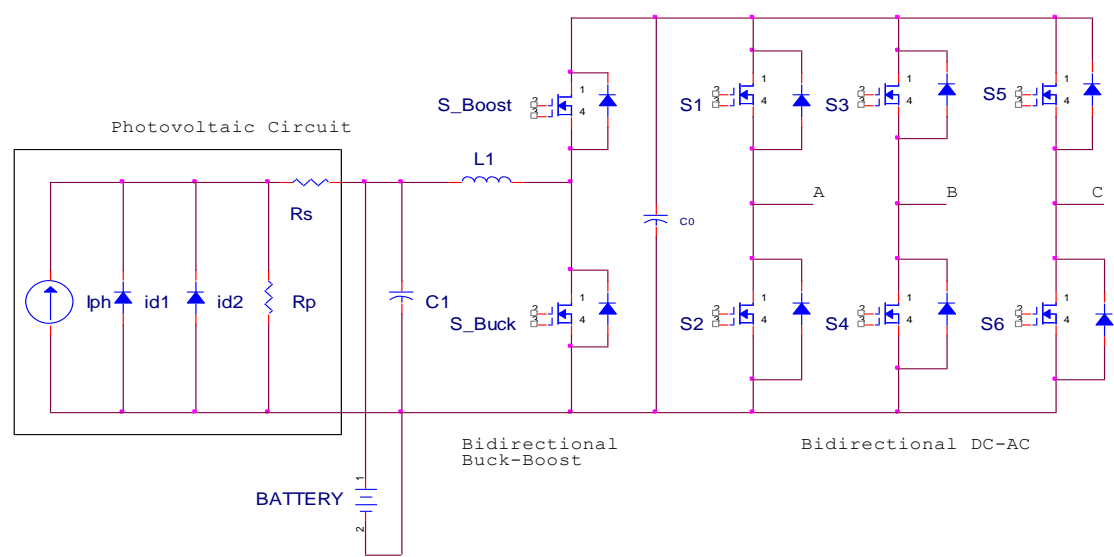

Figure 2. Circuit diagram of the PV fed BDC converter with grid

\section{Bidirectional Converter:}

In this proposed converter is suggested would limit that switching equipment and decrease the THD. Then those single based inductor DC- DC buck-boost converters are used to increase that power factor by a multipleswitching. Then DC-AC bidirectional inverter is used specifically for circuit balancing. In bidirectional converters, and hybrid electric cars, in distribution system, electrical aircraft along continuous with power supply are used in a range of applications. There are two switches in the bidirectional converter. Single switch is to uses by the direction of transmission with another for power transmission. The proposed converter is transferred electricity from that supply to the grid along with effective grid into the supply using the battery serial switch and a series link between the photovoltaic along bidirectional converters endures given with another switch. Figure 2 shows the bidirectional converter circuit diagram is shown. The PV input given supplies into the bidirectional converter into that grid-connected network. Then the single switch would be triggered if the battery is used as the source, leading both forward and backwards.

\section{Modes of Operation}

There are many modes for running the planned bidirectional grid connected converter. Here supply by that planned circuit endures the photovoltaic is supplied by a grid-bundled converter in mode 1 . The S1 switch has disabled along with S2 is activated, and then inductor charge along with the current passes from the PV to the $\mathrm{S} 2$ bidirectional inverter in the front direction.

The S2 switch endures activated along with the S1 switch turns off with effective power flows between battery and the grid throughout effective proposed inverter. PV is isolated ahead circuit suggested. Storage system like battery endures to used and discharged can root by the planned system in mode 2 . The PV endures removed ahead the two-way system. Total grid acts likes supplies along the reverse path of the two-way inverter feature. The current passes between grid and storage. Then the battery is charged along uses by external purpose. Conversely, due to the grid supply input the bidirectional inverter acted as a correction method. When the inverter rectifies, the pulses of the proposed inverter endures to null. Here DC is turned into a BDC and saved on the battery. So, during this mode, the battery charges.

\section{Controller}

\section{PID}

PID consists in general by three signals are proportional, the integrals along derivatives, and is applied to the plant model to provide an error correction signal. Proportional gain along with integral benefit (Ki), derivative gain $(\mathrm{Kd})$ are the profits of each signal. Error is the input of the PID handler and error is effective output of the PID. The math equation is defined by,

$$
P I D_{\text {output }}=K_{p} * \text { error }+K_{i} * \int \text { error }^{*} d t+K_{d} * \frac{d_{\text {error }}}{d t}
$$

\section{Fuzzy}

Either the error input to the PID handler, and the error input to the PID controller. The mathematical equation is presented with. Fuzzy used the FLC method that is one of human and specialist information combinations. The functioning of the expert system is tremendous in terms of Boolean normality. 


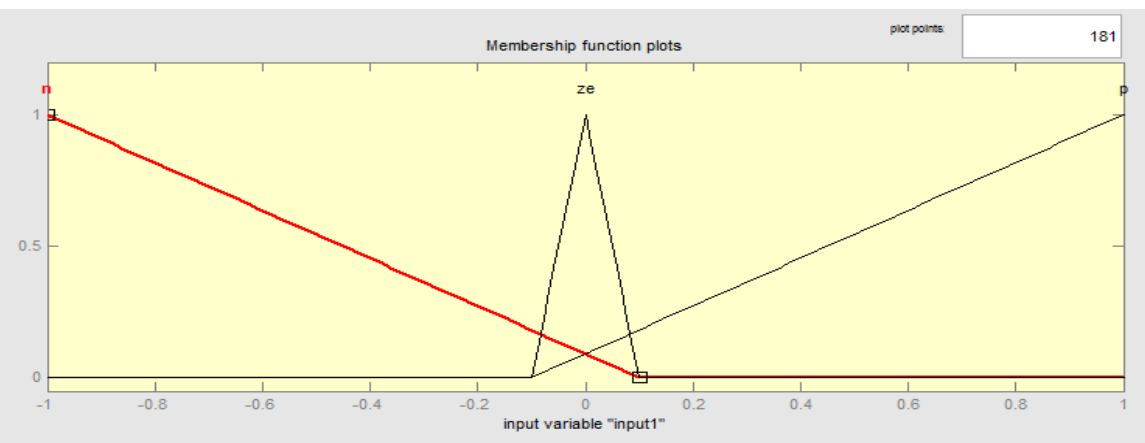

Figure 3. membership function Input variable 1

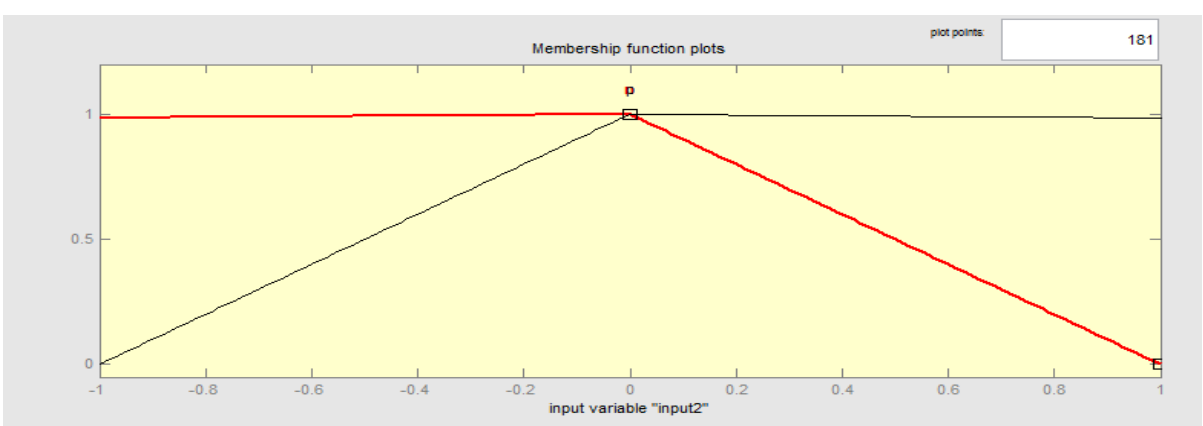

Figure 4. membership function of Input variables 2

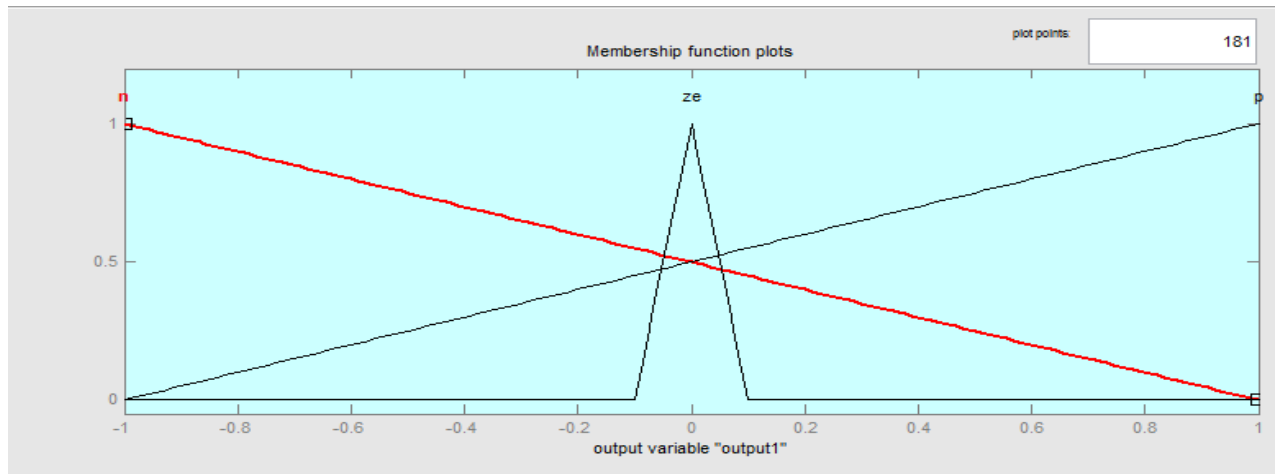

\section{Fuzzy PID}

Figure 5. Membership function of Output variable

The combination of fuzzy-PID is proposed in this paper for voltage regulation of BDC fed grid connected system. In this combination, separate papers were suggested. Dynamic response is expected for the Fuzzy controller. The PI controller provides an interval of reaction time which assures a strong continuous reaction. Both fuzzy and PI controls are used because of these advantages. The amplifier behaviour which saturates the overflow takes place during this multiplicating process. The fuzzy amplifies the PID output and sends the amplified signal to the device model. The equilibrium is decided by,

$$
\text { Fuzzy }- \text { PID }{ }_{\text {out }}=\text { PID }{ }_{\text {out }} * \text { Fuzzy }_{\text {out }}
$$

Due to the less overflow and settlement time than the additional fuzzy PID, the fuzzy-PID with Multiplication operation is applied here. The proposed controller block diagram is shown in figure 6 . 


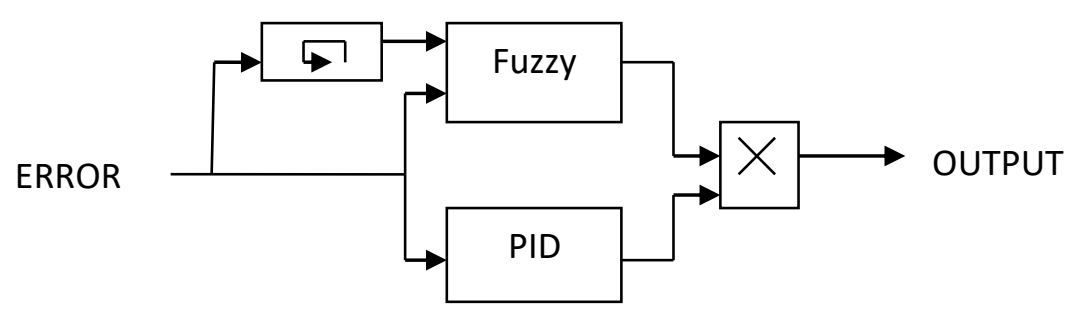

Figure 6. Proposed controller block diagram

\section{Simulation Results}

Bidirectional grid-connected device configuration based on renewable energy has been deployed and findings in MATLAB / SIMULINK have been verified.

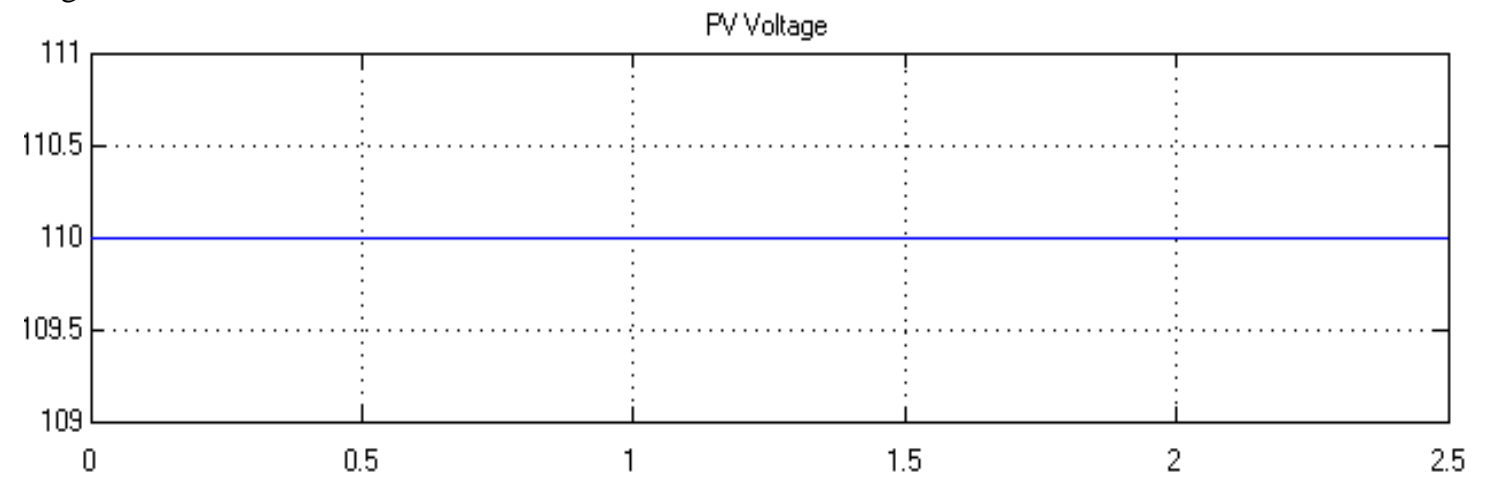

Figure 7. PV Voltage waveform

The control of power photovoltaic age dependent on bidirectional inverter took care of network application is dissected and confirmed with consequences of Fuzzy-PID control with PWM strategies under nonlinear burden condition. The PV yield voltage waveform is appeared in Figure 7. The PV yield current waveform is appeared in Figure 8.

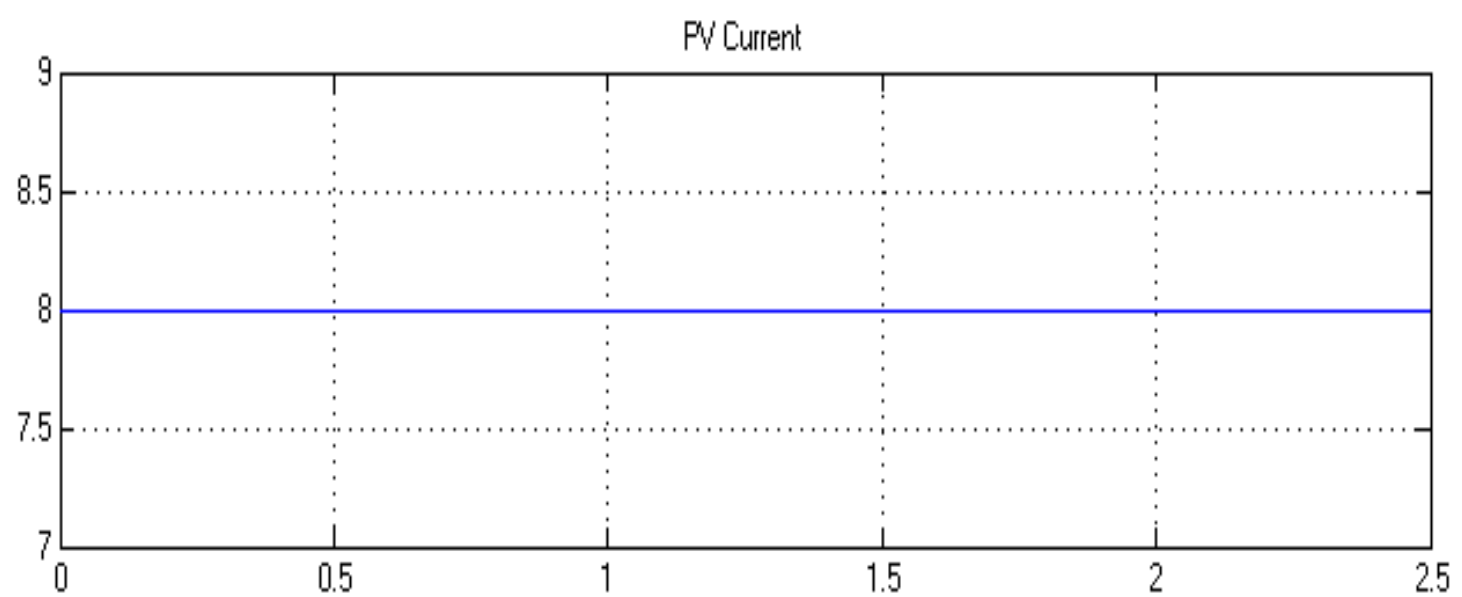

Figure 8. PV current waveform

The charge of battery systems is shown in Figure 9. It can observe that time at 0-1s used as a sources along the proposed converters acts as that forward orientation of effective photovoltaic. The battery time is 1-1.4 and the two-way converter has been working in the direction of the forward. Next the at 1.14-2.5 that grid serves is sources function and the reverse flow path is supplied by the BDC converter. Storage system battery discharged the power supplies along with the two-way inverter-fed charge system in the forwarding direction.

The Battery charging is in the charges opposite direction. Figure 10 displays effective DC link voltages shape by the proposed converter system. Finally figure 11 displays effective two-way inverter voltage waveform. The current waveforms by the proposal inverter are Figure 12 shown in. Figure 13 shows the absolute harmonic distortion. 


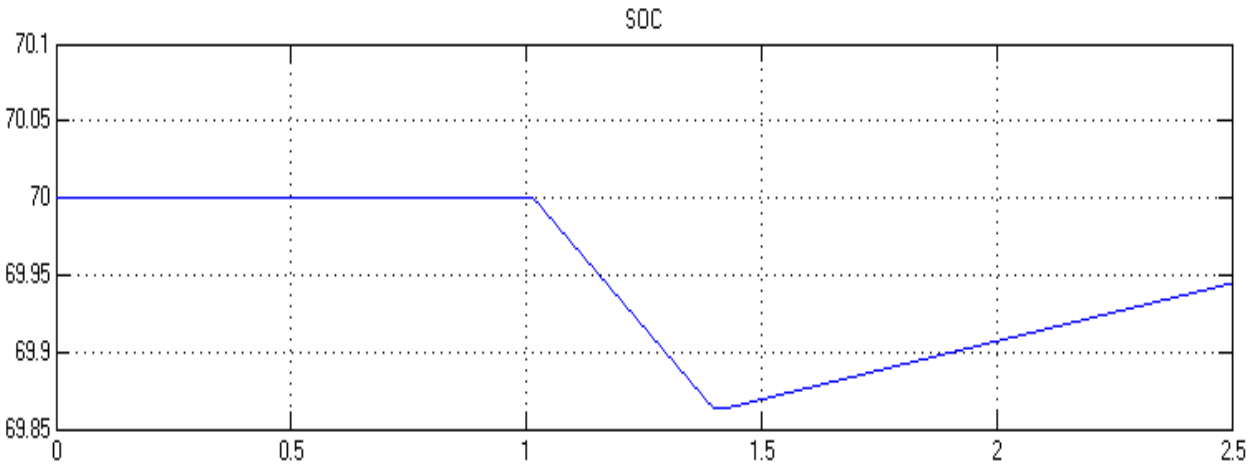

Figure 9. Battery charge condition

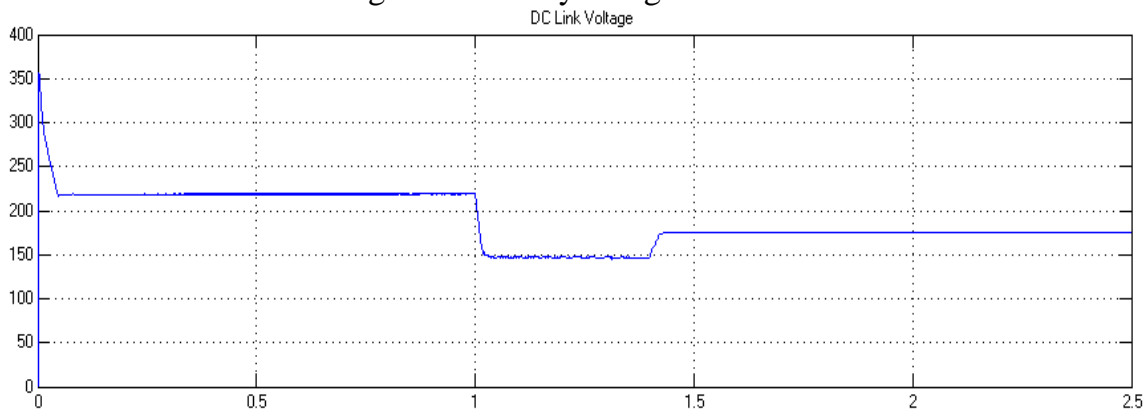

Figure 10. DC voltage Link

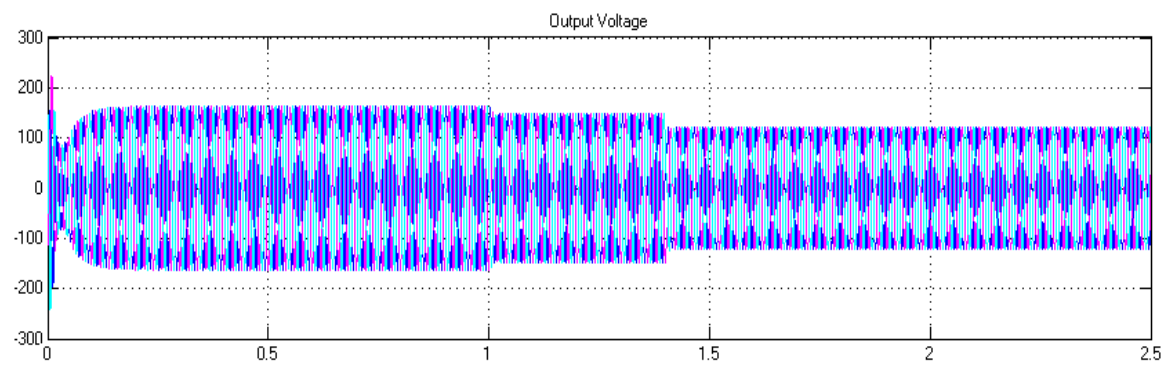

Figure 11. Bidirectional inverter waveforms Output voltage

Output Current

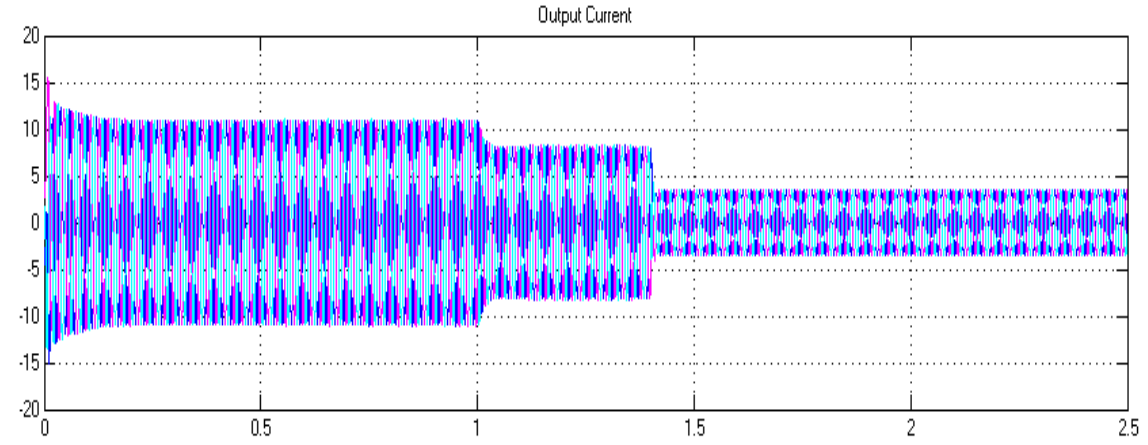

Figure 12. bidirectional Inverter Output current waveformS

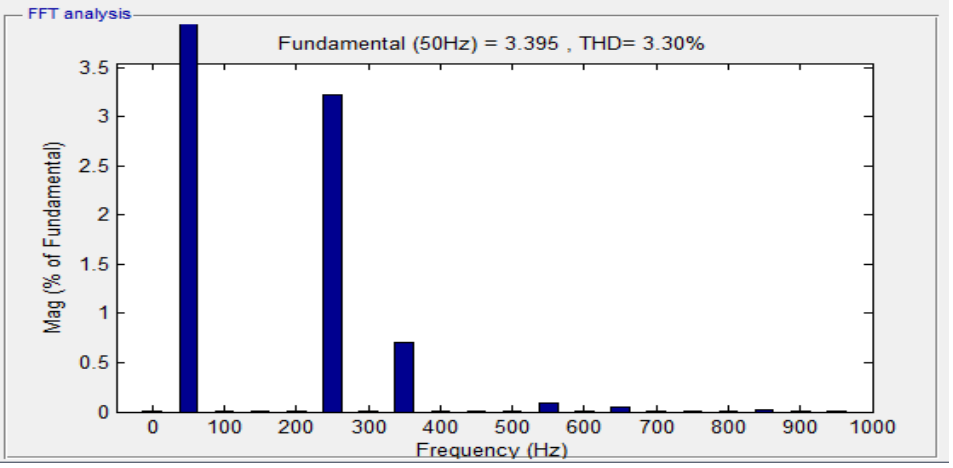

Figure 13. Total Harmonic Distortion (THD) 


\section{CONCLUSION}

Then the artificial intelligence-based proposed inverter (BDC) was developed and introduced (Fuzzy Logic-PID controller). The power flows between PV along with grid is regulated by effective grid-connected system. The BDC converter operations have a stimulation converter in the forwarding direction, and power is supplied to the BDC to stabilise the power and increase system performance. Effective photovoltaic system within a battery power storages system is important by the BDC utility grid interface application not just to monitor and handle electricity demand through the power grid in the future. In reverse, while the photovoltaic device is not available, a battery is charged in order to demand power in the various fields. In effective gridbased BDC inverters PV system, performance and power flow control are controlled and verified through MATLAB/Simulink.

\section{References}

Østergaard, P.A., Johannsen, R.M. and Duic, N., 2020. Sustainable development using renewable energy systems. International Journal of Sustainable Energy Planning and Management, 29, pp.1-6.

Badal, F.R., Das, P., Sarker, S.K. and Das, S.K., 2019. A survey on control issues in renewable energy integration and microgrid. Protection and Control of Modern Power Systems, 4(1), p.8.

Al-Ghussain, L., Samu, R., Taylan, O. and Fahrioglu, M., 2020. Sizing renewable energy systems with energy storage systems in microgrids for maximum cost-efficient utilization of renewable energy resources. Sustainable Cities and Society, 55, p.102059.

Gomathi, S., Suganyadevi, M.V., Kamalakannan, S., Kumar, K.R. and Deepak, A., 2020, September. Active Bridges Based Bidirectional DC-DC Converter for Solar PV application. In 2020 International Conference on Smart Electronics and Communication (ICOSEC) (pp. 1087-1094). IEEE.

Sujitha, N. and Krithiga, S., 2019. Grid tied PV-Electric Vehicle Battery Charger using Bidirectional Converter. International Journal of Renewable Energy Research (IJRER), 9(4), pp.1873-1881.

Bhagiya, R.D. and Patel, R.M., 2019, December. PWM based Double loop PI Control of a Bidirectional DC-DC Converter in a Standalone PV/Battery DC Power System. In 2019 IEEE 16th India Council International Conference (INDICON) (pp. 1-4). IEEE.

Shubhra, S. and Singh, B., 2019. Three-Phase Grid-Interactive Solar PV-Battery Microgrid Control Based on Normalized Gradient Adaptive Regularization Factor Neural Filter. IEEE Transactions on Industrial Informatics, 16(4), pp.2301-2314.

Chatterjee, A., Bhaumik, S., Naskar, A., Mondal, N., Chatterjee, D. and Ray, S., 2020, February. A Transformer-less Grid Interactive Converter Topology for PV based Micro Generator. In 2020 IEEE Calcutta Conference (CALCON) (pp. 497-501). IEEE.

Maity, S., Ghosh, S., Pal, R., Saha, S., Samanta, S., Guha, S., Mondal, R., Sau, R., Pan, S., Das, A. and Maity, J., 2019, April. Performance Analysis of Fuzzy Logic Controlled DC-DC Converters. In 2019 International Conference on Communication and Signal Processing (ICCSP) (pp. 0165-0171). IEEE.

Trujillo, O.A., Toro-García, N. and Hoyos, F.E., 2019. PID controller using rapid control prototyping techniques. International Journal of Electrical \& Computer Engineering (2088-8708), 9(3).

Khather, S.I. and Ibrahim, M.A., 2020. Modeling and simulation of SEPIC controlled converter using PID controller. International Journal of Power Electronics and Drive Systems, 11(2), p.833.

Subroto, R.K., Ardhenta, L., Hasanah, R.N. and Sasminto, K., 2020, July. Fuzzy logic-based tuning of PID controller for zeta converter regulation. In Journal of Physics: Conference Series (Vol. 1595, No. 1, p. 012035). IOP Publishing.

Chao, C.T., Sutarna, N., Chiou, J.S. and Wang, C.J., 2019. An optimal fuzzy PID controller design based on conventional PID control and nonlinear factors. Applied Sciences, 9(6), p.1224.

Kusmantoro, A., Purnomo, M.H., Priyadi, A. and Putri, V.L.B., 2019, October. Fuzzy-PID Controller On MPPT PV To Stabilize DC Bus Voltage. In 2019 International Conference on Technologies and Policies in Electric Power \& Energy (pp. 1-6). IEEE. 